school in - which was equivalent to a three-year course in the high school of this city.

"Supt. of - School from 1894 to $1896 . "$

"Dr. —, Chicago, Ill., Dear Sir:

"Mr. — , the bearer, impresses me as a young man in every rense qualified to take at dental course.

"He states that he completed the graded-school course and a portion of tiee first vear of the high school course in the city of - Since thell be has had several years of practical experience in a telegraph office in this city. "Respectfully,

"Asst. State Superintendent.."

\section{POST-OPERATIVE HEMORRHAGE.*}

\section{A. H. CORDIER, M.D.}

President of the Mississippi Valley Medical Association: Frofessor of Abdominal Surgery, University Medical College.

KANSAS CITY, MO.

I know of no condition in my work that is more horrifying than to realize that a patient upon whom $I$ have just performed an abdominal operation is bleeding dangerously. I know of no condition under which the surgeon will more quickly realize the importance of his work and appreciate more fully the great responsibility existing in every abdominal operation.

An avoidable accident to a patient that may result in the death of that patient is a very serious matter and should not be passed over as a trifling affair by the surgeon. Trying to give contentment to the sorrowing family and friends by assuring them that the all-wise Providence willed it thus may satisfy the unsuspecting and confiding family circle; but does it relieve the mind of the surgeon or save the patient?

The use of unstable ligature material on large pedicles and in localities where the same is quickly absorbed has cost quite a number of patients their lives from postoperative bleeding. It has been my misfortune to lose one patient from a post-operative hemorrhage, the cause being the use of a catgut ligature. After a large series of cases in which I used silk ( $I$ use it altogether now) without an accident of this character, I was persuaded to use catgut in a simple and easy case. This case was in a private residence in a distant city. Three hours after the operation my patient died from hemorrhage, the result of a slipped catgut ligature. I know that the ligature was responsible for this death. It should have been avoided. Dispensations of Providence are garments that may be used to hide the naked truths from an ever-credulous public, but before the eyes of the conscientious surgeon he sees the slanderous injustice of hiding his blunders under such sacred raiment.

That many fatal post-operative hemorrhages are unavoidable no one will deny, and it has been the experience of almost every surgeon to have had such. The recognition of the possibility of a patient's bleeding to death following an operation should only make the surgeon more careful to avoid it and to resort to the best method of speedily checking it when it has taken place.

There is no condition in which quick surgery is more essential; I mean by quick surgery, not alone getting at the work quickly, but rapid work after the secondary operation has begun. A patient who a few hours previously has gone through a severe operation, probably of long duration, or of a character to produce shock, is

* Read in the Section on Obstetries and Diseases of Women, at the Fifty-second Annual Meeting of the American Medical Association, held at St. Iaus, IInn., June 4-7, 1901 . not a very good subject to be given an anesthetic and undergo a reopening of the peritoneum. If the surgeon does not recognize this, the majority of his patients who are reoperated upon will dic. No chronic or deliberate surgery can be tolerated by such a patient.

The use of the Trendelenburg position, while it enables the surgeon to see the bleeding points and to tie them most efficiently, may by gravity, in a patient with a weakened heart, keep a vessel from bleeding during the operation that may bleed profusely on the patient's assuming the horizontal position when placed in bed. This is especially true of capillary oozing from adhesions.

The drainage, as has been urged by Price and others, is not only valuable as a sentinel, but has a direct hemostatic power. All recognize the necessity of a clot, firm in character, to control oozing or even bleeding from larger vessels. Blood in the air-tight peritoneum, in the presence of a peritoneal temperature and abundance of lymph, will not coagulate quickly or at all firmly, as all will agree who have opened the peritoneum in cases of ruptured tubal-gestation or other conditions where there has been a free intraperitoneal bleeding.

A drainage tube in suspected cases for a few hours will do no harm and will surely give the indications of a hemorrhage. If the bleeding is from small vessels, the keeping of the peritoneum dry will be a potent factor in controlling the bleeding. Of course, the tube should not be used unless some reason is present for its use.

While the tube is of much use in suspected cases, its utility is lost in the case where a hemorrhage occurs in a patient in which such an accident is not expected. 'Thus, my three post-operative hemorrhages have been in easy cases where such was least expected. My other secondary operations for bleeding have been in patients of other surgeons. I was called in their absence, saving the patients in each instance.

The symptoms of a concealed hemorrhage are very much like those attending shock, and, if the symptoms alone were relied upon to make a diagnosis, many errors would result. The pulse is increased in frequency in both, and diminished in force equally in the two conditions. There is a sighing respiration in each, the features are pinched and the surface is blanched in the two, the skin is clammy in both, the patient is comfortable and indifferent in each, the pupils are dilated and respond slowly in either conaition.

How is the diagnosis best made? Take, for instance, a fairly strong patient, remove an ovarian tumor from that patient, occupying from fifteen to twenty minutes in the operation. There are no adhesions, there is a small pedicle, a silk ligature is used, and the patient is put to bed in good condition at 9 a. m., with the pulse 80 , the temperature $98 \mathrm{~F}$., and with no shock. Now visit this patient at $3 \mathrm{p}$. m. the same day, and find her with pupils dilated, features pinched, free from pain, the skin white as marble, the lips shrunken and pale, the pulse 160 and very compressible, with a cold, clammy perspiration, all symptoms of shock or hemorrhage. Analyze the history of this case, and experience will say that a patient leaving the table in the condition of this patient, having had no evidence of shock or anything done to produce shock, has an internal bleeding from a broken or slipped ligature; the doubt, if any existed, should be quickly dispelled by cutting a suture and introducing a tube or an artery forcep.

Take another case: Extensive bowel adhesions have been separated in enucleating a growth, the operation is 
a very long one, the patient is more or less shocked at the completion of the operation, this condition continues until it assumes an alarming state, and the question arises: Is this a continuance of the shock, or is it in part, or altogether, due to a bleeding? If a tube had been introduced, no doubt need exist, but in the absence of the tube there is nothing absolutely reliable to guide us but the cutting of a suture and opening a small portion of the wound. This can be done with absolute safety.

On the other hand, if this patient had left the table in good condition and a few hours later presented the shock-hemorrhage picture, it would be quite safe to diagnosticate hemorrhage and act accordingly. As a rule, the first evidences of shock begin during the operation, and many cases of secondary shock in reality are cases of post-operative hemorrhage. Each case is an individual; the experience of the operator and his knowledge of what was done, and who did it, will lend mueh aid in determining the cause of the symptoms manifested, as well as the probable location of the same, if from a bleeding surface or vessel.

If convinced that the patient is bleeding and that the loss of blood will prove fatal, the surgeon's only duty is plain, and he should not shirk it, while giving strychnin, using hot applications, etc. The patient is not safe until the bleeding is controlled. I do not mean a slight oozing such as we often see in the cuve in adhesion cases, but by hemorrhage a loss of blood that quickly shows its dangers by the rapid failure of the patient's vital forces.

It has been my misfortune to lose one patient operated on from a hemorrhage corning on a few hours after ligating a small pedicle with catgut. The abdomen was reopened, but the patient was dead or had ceased breathing when I had begun. The abdomen was full of blood. $I$ have reopened the abdomen in two of my cases besides this fatal one; in both large quantities of blood were lost, but in both recovery followed. In two cases in the practice of other surgeons I reopened the abdomen, saving both patients.

The proper use of the deci-normal saline solution in restoring patients in cases of shock and loss of blood is one of the most valuable additions to our work within the last few years. Its application is so simple and easy and its powers for good are so large that $I$ am surprised that it is not more extensively employed by the profession. The introduction of air into the vein at time of infusion shonld be avoided, yet the danger from this source is not so great as is generally supposed. In one of my cases a faulty apparatus permitted a large amount of air to enter the vein. This was done by one of the nurses handling the bulb of the apparatus in such a manner that it let in air. Not a symptom was produced by this accident. I have in all my cases, save this one, used an ordinary fountain syringe and the cannula of an aspirating outfit or, as in one case, the glass nozzle of an ordinary medicine dropper. Everything ahout the apparatus must be carefully sterilized. The salt should be baked anci the water, when possible, filtered and always boiled. The height of the fountain will govern the pressure. Usually a 3-foot pressure is all that is necessary. The fluid must be kept at a nniform temperature of $112 \mathrm{~F}$. in the bag. A lower temperature will frequently cause the patient to "chill." I think it best to introduce the fluid in two sittings where a large amount is necessary. About 90 grains of common salt to a quart of water is approximately a "deci-normal saline solution."
CONCLUSIONS.

1. In diagnosticating post-operative hemorrhage the operative history will aid much.

2. The symptoms of shock and those of hemorrhage are very similar.

3. In suspected cases the cutting of a single stitch in the incision will tell.

4. The surgery must be quick and decisive in these cases.

5. In cases in which bleeding is expected the tube should be used.

6. Large quantities of deci-normal saline solution will save many patients. This should be used both per rectum and by injection into the veins.

7. Strychnin, belladonna, etc., will not control bleeding from a uterine or ovarian artery any better than from any other artery.

8. The surgeon should do what his surgical conscience tells him is right. Late researches in hematology make it appear that an internal concealed hemorrhage may be demonstrated by a careful blood-count. This, it is stated, will show a decrease in the red cells and an increase in the white.

Very similar symptoms accompany shock from various causes, sisch as internal hernia, etc., none of which produce a change in the red cells. If an operation was performed for the relief of an inflammatory process, this test would lose its value in part, as there would exist at the time of operating a leucocytosis. Saline infusions apparently increase the white cells at first.

\section{DISCESSION}

DR. L. S. MCMURTRY, Louisville.-In my brief remarks I shall allude to the prevention, diagnosis, and treatment of this condition. We all recognize that post-operative hemorrhage is due to either a broken or a slipped ligature. In my judgment, it is rarely due to the former, and almost invariably due to the latter. I must disagree with the essayist that the ligature material used has much to do with the causation of hemorrhage. I myself, like the essayist, prefer silk, but I recognize the fact that there are gentlemen present who use catgut uniformly, and they can show just as few instances of post-operative hemorrhage as can those who use silk exclusively. After all, it is not so much the material used as the method of application. The man who is in the habit of using one kind of ligature gets accustomed to it and applies that ligature with more celerity and security than if he changes his method frequently. A man who is in the habit of applying catgut co a pedicle or vessels will succeed just as well as he would with silk. Care must be observed in the application of a ligature. For example, in the operation for the removal of uterine fibromata the broad ligaments are usually in a state of great tension, and if the ligatures are then applied, and divided, you are inviting the accident of slipped ligatures because just as soon as you relieve the tension, the tissue shrinks and the ligament is apt to slip out of the grasp of the ligature. This accident can be prevented by securing the broad ligament with clamps, and then when there is no tension apply the ligature, place it carefully home, and make sure that it is of good and tested material. As to the prevention of this accident, then, it depends for the most part on the care and surgical precision with which the ligature is applied.

The diagnosis is to me a matter of great difficulty, and the importance of it can not possibly be exaggerated. Secondary operations in the abdomen are fraught with the greatest peril. A patient who has had an operation performed on one day and is obliged to submit to another on the next day can not be expected to bear up under the severe strain and shock. These patients will usually collapse and die, even though the post-operative hemorrliage, 
for which the operation is performed, may have been very slight. You can take a woman with a ruptured tubal pregnancy and with the abdomen full of blood, open the abdomen, occupying nearly an hour, perhaps, in doing so, and she will not have the same amount of shock. The question. of interference for the prevention of post-operative hemorrhage is the most difficeult, in my judgment, that the surgeon has to decide. Lives have been lost by deciding to do it, and lives have been lost by deciding not to do it. The question must be decided with great care and deliberation. In neurotic women, the cireulation and respiration are markedly increased in rapidity after abdominal opera. tions, and it will test the judgment of the most experienced surgeon. Jatients with rapid pulse, sighing respiration, neurotic temperament, exhibiting the symptoms the resuit of neurotic influences, will so simulate post-operative hemorrhage that it will require the greatest eare and judgment when making a diagnosis. Therefore, it must not be decided hastily.

The intravenous injection of normal salt solution should never be done when it is possible that the hemorrhage is still going on, because it will be increased on account of the open vessel. The treatinent should consist in operative inter. ference decided carefully and judiciously, and which should be executed promptly and rapidly. I do not believe with the essayist that the amount of blood lost should be an indication for or against the interference in post-operative hemorrhage. Oftentimes you will find profound collapse and death occurring from the loss of almost an insignificant quantity of blood. On opening the abdomen of a woman who has had one operation which was followed by symptoms of hemorrhage, you will sometimes be surprised to see how little blood has been lost. There may be just oozing from the venous surfaces, and which will lead to disatrous result. I have opened abdomens and found pedicles intact, but on the side of the pelvis where the adhesions were severed, there was a little trickling of venous blood, and the patient almost gone.

Prompt hemostasis must be performed, and you can not expect to tie vessels when there is oozing, but arrest hemorrhage by quickly packing the entire cavity with gauze. There is no procedure in this department of surgery that requires the exercise of such good judgment and judicious action as is required in the treatment of post-operative hemorrhage.

Dr. Seth C. Gordon, Portland, Maine-Since 1884 I have used no suture or ligature except eatgut, with the single exception of silkworm gut, for the closure of abdominal wounds. I believe the time is coming when we are all going to use absorbable sutures and ligatures. In $\mathrm{my}$ own experience, I still have to see the first intraperitoneal hemorrhage. I never tie off the pedicle without using a needle, threading one end of a long ligature and carrying it through the pedicle below the ligature, then double-wrapping and firmly tying it. In all cases of hysterectomy, where I am to remove everything, I ligature with the needle as described. I put on the clamp and commence my first ligature by tying the ovarian artery tightly, then carry my needle below that, make another knot, which prevents hemorrhage. The rest is the same, over and over, down to where I cut off the uterus. It is all done with one suture. I certainly would not use a drainage tube in order that 1 might be protected against pelvic hemorrhage.

Dr. Howard A. Kelly, Baltimore-I am very glad to hear this timely subject brought up for discussion. My former associate in the Johns Hopkins Hospital, Dr. Clark, who is here, will probably recall a case we had some six years ago, where eight days after the operation a woman died from hemorrhage from the right uterine artery, which autopsy showed to be due to the absorption of catgut. Since that time I have preferred to use absorbable ligatures upon what I call cardinal vessels, the two ovarian and uterine arteries. Furthermore, I see no reason why we should desire any other material than fine silk aseptically used; with that, and with gloves worn at the operation, the ligatures will never trouble you afterwards. It is of extreme importance in this work to avoid the antiquated method of tying the pedicle by a ligature which transfixes it and is then tied both ways. The proper ligation of the ovarian pedicle is one ligature at the brim of the pelvis, and the other at the uterine cornu. It is as a rule best to put two ligatures upon all the principal vessels. The most important step of all in the avoidance of hemorrhage is the thorough systematic review of the entire field of operation before closing the abdomen, looking over all ties, and pronouncing a definite judgment on each step of the operation.

The best assurance that there is not going to be any hemorrhage lies in the satisfactory conduction of the operation, so that when the patient lies later in a collapsed condition, and the assistant or nurse is rather doubtful as to whether there is any hemorrhage, the operator ean say with positive assurance that he knows there is none. The operator and his assistants ought to inspect the patient carefully as she leaves the table; to examine the pulse and the mucosæ, to get an exact idea of her condition, and in order to make comparisons later to determine whether much blood has been lost. If the operator is unable to say whether or not there is hemorrhage, open up the patient at once, and see. If the patient is a collapsed neurotic, it will not nurt her; but if she has hemorrhage, prompt action will save life.

I give salt solution under the breasts, and as the patient goes onto the table. Do not stop to pick out the exact seat of the hemorrhage. You know which vessels it may come from; therefore, go down into the pelvis with fingers and clamps, eatch them all, both uterine and ovarian, then clean out the blood and scarch for the particular source of the hemorrhage.

Dr. Henry O. MarCy, Boston-I believe that hemorrhage is usually the fault of defective technique. First, the vessels should be sought for and ligated. Then, it is important to suture the tissues over the vessels of these larger wounds so as to have additional control nver the ligatures. I earnestly advise the folding of the peritoneum over the stump, as it also prevents hemorrhage, and the possible subsequent adhesions, which otherwise may result. I emphasize this from the standpoint of experience. I believe my best contribution to surgery is the buried animal suture, which $\mathrm{I}$ first employed in 1870, and have used it almost daily since. Until 1882 I used only eatgut, and with reasonably satisfactory results, but in the long experimental studies that I made on animals, I found that catgut was absorbed much too rapidly, and that the knot is not satisfactory. Then I introduced the tendon suture, as a substitute. which I consider in every way superior.

I take issue with Dr. Kelly in regard to the rubber gloves and the silk, as they do not always prevent the condition he speaks of. I have often found that silk ligatures give rise to irritation and serious subsequent trouble. I am convinced that we should use only absorbable ligatures and sutures, and in such a way that we may be doubly sure that we have control of hemorrhage before the patient leaves the table. We should intrafold all abraded surfaces in pelvic or abdominal wounds, so that we may not have the condition Dr. McMurtry referred to, namely, oozing surfaces. Faulty technique is undoubtedly the most frequent cause for the occurrence of complications of any kind. I have used buried animal sutures in fully three thousand cases of abdominal operations, and I assure you that I have yet to see any reason why I should not be more than ever an enthusiastic advocate of their general adoption in all aseptic surgery.

Dr. Frederick Holme Wiggin, New York City-I have found chromicized eatgut No. 1, or No. 2, to be a much more satisfactory material for ligature or suture than silk. In former years much trouble frequently resulted from the use of silk for this purpose, not only in my own work, but in that of others. Frequently, in the course of my service at the City Hospital in New York, in past years, I have had occasion to remove silk ligatures and sutures employed by other surgeons at previous operations, but at the present time, since the use of catgut for suture and ligature material has become to be more generally used, these operations are becoming infrequent. If chromicized catgut is used, taken direct from the alcohol in which it is preserved, there should be no trouble 
in its being absorbed too soon or of its infecting the tissues. It has been my practice, whenever possible, to pass ligatures around the vessels to be tied by the aid of a needle, and when this is done and the catgut properly tied, there is no possibility, in my opinion, of their slipping. One of the causes why ligatures of eatgut or other material slip is that there is too much tension applied to the parts when the ligature is being tied. Another reason why operators of ten fear to use catgut ligatures is because they find when they tie them the knot frequently comes undone. This is because they do not understand how to make the knot. They tie the catgut lizature as they would sne of silk, namely, with a double loup first, followed by a single one. When the knot is tied in this way, it will very often become undone. Some years ago, Dr. C. C. Fredcrick, of Buffalo, called $m y$ attention to the fact that catgut, tied first with a single knot and then with a double surgical knot, the ends of the catgut being left reasonably long, would never slip. Since that time I have used catgut for ligatures en tirely in my work, and have had no reason to regret its use.

The surgeon should aim, in my opinion, to tie the vessels as directly as possible, including as little other tissue as is absolutely necessary.

I would like to call attention to the following important point: Where abdominal operations are performed on patients in the Trendelenburg posture often when the operation is completed there is apparently no hemorrhage, but in many cases when the patient is lowered into the horizontal position, you will find on looking about the pelvis, bleeding points. I have often found that if I had not been careful in looking over the field of operation after the patient had been lowered into a horizontal position, I would probably have lost the patient from hemorrhage.

I believe that when catgut is probably used, there is no danger of hemorrhage oceurring from vessels tied off with it.

Dr. C. C. Frederick, Buffalo-I have used catgut exclusively for $t \in n$ years, and in nearly a thousand cases, and I had only one case of slip ligature. That was in a vaginal hysterectomy where the pedicle had been pulled down a great deal during the operation. I believe that was the cause for the ligature slipping.

As to the point made by Dr. Kelly, that the catgut absorbs too rapidly, and that the plug occluding the vessel may be extruded by arterial pressure, and then have hemorrhage, I think that possibly may be true. Formerly, the catgut was sterilized by the Cumol method or alcohol under pressure, being raised to a temperature of 212 and above, rendering it rapidly absorbable. With the modern method of sterilizing, however, I am positive that the eatgut is rendered more durable in the tissues. I refer to the formalin process. I know positively that the catgut so prepared is not absorbed until two or three weeks afterwards, hence Dr. Kelly's objection to it eannot be sustainerl.

Insure against slipping of the ligature by drawing the first knot up tight, and then make the double knot. Do not cut the ends of your ligature too short. There is absolutely no objection to leaving ore-half or even three-quarters of an inch of catgut. It aids in preventing the slipping. That is the method $I$ have followed in all my cases.

Dr. M. L. Hariss, Chicago-I wish to enter my protest against the ligation cn masse of large pedicles. 'There is scarcely a place nor tine in abdominal or pelvic surgery where this is necessary. In most cases where secondary hemorrhage has occurred it will be found that the ligature has been applied to the pedicle en masse. We should invariably follow the sound surgical rule of applying the ligature directly to the artery or the bleeding vessel. If we ligate en masse, the tissue about the vessel may shrink, releasing the vessel, and hemorrhage is sure to follow. In all cases where there is a large mass which bleeds, this should always be closed by a suture, passed with a needle, and in a continuous manner. This will prevent not only the hemorrhage from the vessels, but also the oozing. I think there is no time or place in abdominal or pelvic surgery in which the mass ligature should be applied.

Dr. G. B. Massey, Philadelphia-It seems to me that one side of this question has been somewhat neglected, namely, the future efrect on the patient of the material used as a suture. The question of secondary hemorrhage brings up that of the relative value of absorbable and non-absorbable suture materials, but the discussion has been neglected on that side of the question which comes elosest home to those of us who are not surgeons, and who see these cases after they leave the surgeon, sometimes years afterwards. I allude to cases of socalled post-operative neuroses. I have seen many of them, and have one under treatment now, in which the suffering scems to have been caused by some irritable focus situated in the uterus, which is partly removed. There is a tender mass in the situation of the remainder of this organ about the size of half a walnut. This patient was operated on six years ago, and since then she has been obliged to spend her mornings recumbent. I do not know what kind of sutures were used, and I merely wish to emphasize that there must be some reason why the surgeons are changing their suture material. I do not believe that non-absorbable material should be left in the body if it can be avoided. Something ought to be used that will disappear by absorption.

Dr. Condrer (closing the discussion)-The principal object of my paper was to call attention to the fact that we must not trust too much to nature in controlling hemorrhage. It is surprising how much we do risk in these cases when we would not think of risking anything in the axilla or popliteal space. Frequently, in abdominal operations, we let oozing go on and trust to nature.

Another point is the question of diagnosis. You find that they will frequently say that the patient was placed back on the table and the incision reopened in order to determine the existence of hemorrhage. This is useless. If the question is whether you have an intraperitoneal hemorrhage, it is necessary to have everything thoroughly sterilized and keep the patient in bed, so as not to disturb her. Clip a stitch and introduce a long sterilized forceps into the wound and detect the hemorrhage. That is the casiest and safest way in which to determine it.

I would never think of using a saline solution while the operation is going on, as the vessels are all widely open and it would simply increase the hemorrhage. However, the hot solution thrown into the rectum is very beneficial in these cases. It matters not what ligature material you use. We always find a diversity of opinion on that subjcet. A number of gentlemen have talked about absorbable material, and each one has a different opinion as to what should be used, and how. We are dealing with an uncertainty, as regards hemorrhage, when we tie a pedicle with catgut ligature. I have seen them absorb in three days, which is certainly too soon. In regard to the mass ligatures, I do not know how we can get around it; we sometimes have a very broad pedicle and time is an important factor. If you want to undertake and divide these tissues, picking out the individual vessels, you will take up a great deal of time. I do not see any reason why we should not use the mass ligature. One of the safe courses in the use of silk is to tie in a mass ligature, so that the structures will overlap the ligature, and the two surfaces above and below come in contact, and the pedicle remain alive. It will get up an anastomotic circulation, and the ligature will remain clean. When you get silk infeeted you can not tell what will become of it. The same applies to chromicized catgut, tendon, etc. I have seen it working cut of a hernia case twelve and fifteen months after the operation, and $I$ am sure that it is less certain than silk. Where you use silk, you do not have to dance around and sec how to use it. Put on a good stout piece of sterilized silk, and in the majority of cases you will have no hemorrhage.

Hemostasis of the Liver.-Baldassari inserted wedgeshaped fragments of decalcified bone between the lips of the wound in the liver, in his experimental research on dogs. The wound was then sutured, including the intermediate bone in the stitches. When the animals were killed later there were no evidences of the slightest oozing of blood or hemorrhage from the wound, and the bone was absorbed, cicatricial tissue taking t; place. The bones were rinsed in an acid, then in 50 per conc. alcohol containing a little sublimate, and finally soaked in a hot saline solution to remove the sublimate and soften them. 